\title{
Écosystème
}

\section{Implants, IA \& art (une méditation)}

\section{David Jhave Johnston}

Volume 3, numéro 1, 2021

Intelligence artificielle

URI : https://id.erudit.org/iderudit/1075895ar

DOI : https://doi.org/10.7202/1075895ar

Aller au sommaire du numéro

\section{Éditeur(s)}

La chambre blanche

\section{ISSN}

2562-3222 (numérique)

Découvrir la revue

\section{Citer cet article}

Johnston, D. J. (2021). Implants, IA \& art (une méditation). Écosystème, 3(1), 55-68. https://doi.org/10.7202/1075895ar
Résumé de l'article

La technologie a rapidement décalé les paramètres du possible, mais l'appareil instinctif intuitif de nos corps auto-orientés tribalistes et émotivement volatiles reste limité à une mentalité de troupeau.

Au cours des 15 prochaines années, l'interactivité vocale et subvocale deviendra accessible au grand public, et les interfaces cerveau-machine filtreront hors des laboratoires pour se glisser dans le cerveau des premiers adoptants.

Interfaces cerveau-machine (IMC) : que vont-elles changer? Cet essai est une exploration et une idéation de quelques-uns des problèmes éthiques potentiels de l'IMC à partir du printemps 2020. d'utilisation que vous pouvez consulter en ligne.

https://apropos.erudit.org/fr/usagers/politique-dutilisation/ 


\section{David Jhave Johnston}

\section{Implants, IA \& art (une méditation)}

Résumé

La technologie a rapidement décalé les paramètres du possible, mais l'appareil instinctif intuitif de nos corps auto-orientés tribalistes et émotivement volatiles reste limité à une mentalité de troupeau.

Au cours des 15 prochaines années, l'interactivité vocale et subvocale deviendra accessible au grand public, et les interfaces cerveau-machine filtreront hors des laboratoires pour se glisser dans le cerveau des premiers adoptants.

Interfaces cerveau-machine (IMC) : que vont-elles changer? Cet essai est une exploration et une idéation de quelquesuns des problèmes éthiques potentiels de l'IMC à partir du printemps 2020. 
«Tout ce que vous touchez, vous le changez. Tout ce que vous changez vous change. La seule vérité durable est le changement. » - Octavia E. Butler, Parable of the Sower (1993)

Les éventuels implants cérébraux entreront dans la phase d'essais sur les humains en $2020 .{ }^{1}$ Ces implants s'appuient sur l'intelligence artificielle pour décoder et coder les pointes neuronales. Qu'est-ce qu'une puce IA dans la tête / cœur signifie pour la pratique de l'art, pour les artistes et les citoyens? Dans le présent essai, j'explore et j'imagine quelques implications (de l'intelligence de la machine intégrée directement dans le cerveau humain) en méditant éthiquement sur cette technologie, et en examinant les changements qui se sont produits dans ma propre pratique artistique en raison de l'IA.

Entre 2004 et 2019, plusieurs changements technologiques ont eu lieu. L'art Web est mort. Les médias sociaux ont vu le jour. Les téléphones cellulaires sont devenus normatifs. L'apprentissage profond a émergé. Les mégadonnées sont devenues massives. Les fausses profondeurs sont devenues réelles. Le partage est devenu idéologique. Et les oligarchies ont commencé à tirer profit de l'intimité en utilisant l'apprentissage automatique.

Quelles avances éthiques avons-nous observé dans l'humanité au cours de 15 dernières années? Dans les 500 dernières? Nos dirigeants sontils devenus supérieurs? Plus calmes? Plus sains? Plus sages? La technologie a rapidement changé les paramètres du possible, mais l'appareil instinctif intuitif de nos corps auto-orientés, tribalistes, émotivement volatiles, limités sur le plan cognitif, reste le même.

Dans les 15 prochaines années, l'interactivité de la voix et de l'expression sousvocale deviendront courantes, les interfaces cerveau-machine n'émaneront qu'au goutte à goutte des laboratoires pour pénétrer le cerveau des premiers adoptants.

\footnotetext{
${ }^{1}$ Mardi le 16 juillet 2019 20:00 heure de l'est Neuralink a annoncé son implant cérébral N1. Ils décrivirent la recherche dans une publication : « Nous avons construit une multitude de petits « fils » d'électrodes flexibles, avec pas moins de 3072 électrodes par tableau répartis sur 96 fils. Nous avons également construit un robot neurochirurgical capable d'insérer six fils (192 électrodes) par minute. Chaque fil peut être inséré individuellement dans le cerveau avec une précision micron pour éviter la vascularisation superficielle et le ciblage des régions spécifiques du cerveau. » Le réseau d'électrodes est emballé dans un petit dispositif implantable qui contient des puces personnalisées pour l'amplification et la numérisation de faible puissance : le dispositif pour 3072 canaux occupe moins de 23 à $18,5 \mathrm{~mm}^{3}$. Un seul câble USB-C fournit des données de pleine bande passante à partir de l'appareil, enregistrant tous les canaux simultanément. Ce système a atteint un rendement de pointe allant jusqu'à $85,5 \%$ pour les électrodes implantées de façon chronique.
} 
J'ai l'intention de recevoir un implant, s'il est sécuritaire, facile à extraire et élaboré de façon collaborative. Je veux qu'il transcrive mes pensées. Qu'il passe au crible ces pensées à la recherche de fragments de langage qui sont significativement radicaux, pour trouver des groupes de mots qui sont des anomalies originales. Je veux un résultat linguistique et je veux être en mesure de pirater le code de mon implant pour l'optimiser en fonction de mon sens de qui est important. Bref, je veux un implant pour trouver la poésie dans mon esprit et l'en extraire.

\section{L’intimité entraîne la vulnérabilité}

N'importe qui avec une puce implantée dans le cerveau - y compris les artistes, les poètes, les rêveurs, les spéculateurs, les imbéciles, et les inventeurs... en fait, tous ceux qui hallucinent des réalités alternatives, rêvent d'utopies, ou suivent émotionnellement d'instables courants d'intention pure - sera en danger. Dans un contexte bénin, l'art impliquera une injection synthétique directe, neurone à neurone, de l'imagination du corps au corps : une expérience partagée, immédiate et viscérale. Subscribe to the artist! Live my life as I live it. (Souscrivez à l'artiste! Vivez ma vie comme je la vis) En même temps, lorsqu'ils seront utilisés par des gouvernements autoritaires, ou dans des filets de contrôle mal intentionnés des consommateurs, les citoyens avec un implant, deviendront des réservoirs en cage, sujets d'un parfait système tyrannique omniscient. Surveillés par des algorithmes imcapables, engourdis par une omniscience déboussolante, incapables de se défaire de la trame qui anticipe leurs pensées.

Ce n'est pas seulement la lecture d'une idée qui est en jeu, c'est son écriture également. Décodant par la zone primaire du cortex qui est lié à la parole, ce que nous pensions dire, notre parole. Pour le meilleur ou pour le pire, il est hypothétiquement possible de réécrire ce que nous pensons avant de le penser, de nous faire penser et dire ce qu'un système (politique/artistique) souhaite. Comme Philip Sabes l'a dit lors du lancement de Neuralink : "Inscrire des informations dans le cerveau : les éléments de base de cette technologie sont déjà là. $\|^{2}$ Prenez la thérapie cognitive activée par l'intelligence artificielle, ou l'implantation augmentée par l'intelligence artificielle des syndromes post-traumatiques, de la schizophrénie, de l'insomnie ou encore utilisée comme traitement des phobies. Imaginez l'avenir des films ou des processus vécus d'immersion en art expérimental. Considérez le soma comme un

${ }^{2}$ Neuralink Launch Event. 2019, fichier numérique, 104 minutes, en ligne, https://www.youtube.com/watch?v=r-vbh3t7WVI\&feature=youtu.be, page consultée le 23/01/2021. 
algorithme, les zombies de l'achat automatisé, et les guerres de propagande où les civils deviennent des robots.

Voici une pensée incontrôlée: le contrôle est en fait ce qu'exercent les artistes. Évoquer ou contraindre les autres à sentir ou à appréhender le savoir d'une nouvelle façon c'est les contrôler. Parfois, le contrôle est volontaire. Parfois réflexif. Un maître-artiste comprend le contrôle. Un artiste de génie en a besoin. Rassemblons-nous tous pour prier de façon méditative pour une évolution éthique de l'humanité.

\section{Qu'est-ce qu'un écart de 15 ans signifie pour l'art?}

En 2004-2005, j'ai produit deux œuvres d'art lors d'une résidence à LA CHAMBRE BLANCHE. Une œuvre (Etay : http://etay.ca) était une installation autonome où j'avais intégré des caméras vidéo et des microphones dans mon atelier d'artiste au centre-ville de Montréal près du quartier Hochelaga-Maisonneuve. L'autre œuvre (Sooth : http://www.glia.ca/SAIC/) était basée sur le Web qui utilisait des volées de séquences poétiques présentes sur la vidéo.

Etay faisait usage d'une sorte système de surveillance du pauvre à l'intérieur du loft où je vivais. Des artistes postulaient pour vivre dans mon espace alors que je vivais à LA CHAMBRE BLANCHE. En échange, ils acceptaient de devenir les sujets de la conscience de la machine. J'avais installé des caméras parfois, incluant dans le miroir de la salle de bain qui était remplacé par un écran tactile pour faire en sorte que le miroir au-dessus du lavabo devienne un système réactif fluide où le doigt pouvait brouiller le visage. D'autres caméras appliquaient une récursion pour que le corps se déplaçant dans l'espace finisse par se superposer avec le temps.

La personne qui vivait dans le loft avait le contrôle sur la façon dont les images de surveillance étaient traitées par les filtres et les applications. Ils expérimentaient sur leur propre extrant perceptuel. Téléchargés sur une application personnalisée sur mon serveur, les résultats ont été remixés à l'intérieur d'un module Flash personnalisé. Dans l'innocence et l'excitation, ce système expérimental de filtres appliqués par l'utilisateur sur la vidéo a anticipé le narcissisme frénétique de Vine ou Instagram. Cela a été construit à l'aide de MaxMSP Jitter et rétrospectivement inspiré par le visionnaire Quiet : We Live in Public de Josh Harris réalisé en 1999. 
À cette époque, l'image rendue par l'ordinateur était restreinte. The Giver of Names de David Rokeby en 1997 a réussi la segmentation d'objets, mais chacun était identifié avec beaucoup moins de précision qu'un téléphone cellulaire n'arrive à le faire maintenant à l'aide d'une lentille Google. Non seulement il existe des systèmes de capteurs consolidés datant de 2019 qui sont utilisés sur les appareils robotiques dans le secteur de l'économie industrielle mondiale, mais l'architecture de l'intelligence artificielle présente sur le nuage(cloud), qui analyse et interprète les flux denses et rapides de données qui émergent de ces fils sensoriels, est en expansion active. ${ }^{3}$

\section{Robo-Muse}

À l'heure actuelle, j'expérimente à l'aide d'un système Azure Kinect qui comporte six microphones dans un tableau circulaire, une caméra de détection de profondeur infrarouge, une paire d'yeux stéréoscopiques, un gyroscope et un accéléromètre. Le kit de développement a été mis sur le marché en juin 2019. Il est destiné à la robotique. Plus précisément la robotique industrielle qui requiert une sorte d'appareil sensoriel qui émule le fonctionnement rudimentaire de l'appareil sensoriel et le cervelet d'un organisme. L'appareil est optimisé pour une suite de " services cognitifs " d'intelligence artificielle basés sur le nuage qui lui permettent de distinguer les corps, de reconnaître les visages, de comprendre le langage et d'interpréter les gestes, ainsi que d'apprendre des tâches et de s'orienter dans un environnement en changement. Essentiellement, il équivaut à un cortex sensoriel rudimentaire. Un cervelet connecté au Web. Indifférent et stupide comme une machine peut l'être, mais rapide, infatigable, et en fait, tel un pore sur la peau d'un robot-quasi-dieu, globalement intelligent.

Dans l'actuel système techno de plateformes de services, des artistes autonomes comme moi, travaillant de façon indépendante sans l'aide d'un énorme budget de recherche, peuvent (en faisant tourner une agglomération de machines d'intelligence artificielle provenant du nuage et louées à la minute) avoir accès à de très puissants GPU and TPU. En accrochant l'apprentissage profond aux innovations robotiques industrielles, il est possible de créer les prototypes anticipés par l'appel au calcul émotionnel de la visionnaire Rosalind Picard ${ }^{4}$, Kismet ${ }^{5}$ cubed.

\footnotetext{
${ }^{3}$ Alphabet et Facebook et Alibaba et Amazon et bien d'autres empilent des stocks massifs de richesse : données et algorithmes. Les traders à haute fréquence déjouent l'esprit humain. L'utilisation primaire de l'apprentissage profond est passée de la recherche altruiste par des pionniers autonomes à une concurrence consolidée d'avantages corporatifs et politiques, de l'exploration ouverte à l'exploitation des capacités d'infrastructure, de l'optimisme techno-utopie à l'anxiété d'extinction anthropique.

${ }^{4}$ Rosalind W. Picard, Affective Computing, Cambridge et Londres, MIT Press, 1997, 306 p.
}

${ }^{5}$ Cynthia L. Breazeal, Designing Sociable Robots, Cambridge et Londres, MIT Press, 2004, 282 p. 


\section{Intelligence artificielle artisanale de petites données}

Pour Sooth en 2005, j'ai programmé des groupes de phrases poétiques qui traversaient au vol des vidéos en ligne. Ces phrases pouvaient être déclenchées par les clics de l'utilisateur et étaient limitées à l'intérieur de régions. Un son fut cartographié pour chaque phrase et reflétait sa position dans l'espace (un déplacement panoramique quand la phrase volait d'un côté à l'autre ; augmentation et diminution de volume en lien avec la taille). J'ai défini tout cela en programmation ; utilisant le code fixe comme paramètres d'un comportement réglementaire. II existait un certain caractère aléatoire inhérent, mais cela a été essentiellement chorégraphié par le code humain et l'interaction de l'utilisateur.

En revanche, en 2019, pour une exposition avec Anteism Press ${ }^{6}$ j'utilise Wekinator, un logiciel d'apprentissage automatique qui transforme les données d'un capteur de caméra infrarouge compacte (Leap Motion) en sortie OSC qui influence les paramètres d'une onde sinusoïque, et contrôle la position de phrases poétiques. J'ai écrit très peu de code pour cela. Les règles sont décidées par les réseaux neuronaux d'apprentissage automatique. Le logiciel utilise de petits lots de données pour s'entraîner. Si vous ne l'avez jamais essayé, il pourrait être un peu difficile de comprendre les petites données, cela ressemble à ce qui est appelé un apprentissage à partir de zéro. Laissezmoi vous donner un exemple concret. Pour que la machine apprenne à cartographier le chemin de l'intrant à l'extrant, elle doit échantillonner l'intrant (dans ce cas, les gestes de la main). Imaginez que je décide que lorsque ma main est levée et ouverte, le son déclenché est clair et aigu ; et quand mon poing est placé au niveau de mon ventre, c'est un son de basse profonde qui est émis. Pendant quelques secondes, j'échantillonne chaque son selon la position de mes mains. Ensuite, les filets neuronaux de Wekinator calculent comment le déplacement entre ces deux positions d'ouverture et de fermeture de ma main qui monte et descend provoque une cascade de variations et d'interpolations transformatrices de cette structure audio.

L'interactivité peut maintenant être apprise par de petites données ingérées par des dispositifs agiles, formés en quelques secondes pour reconnaître les changements dans le format comportemental et les recartographier sur un affichage créatif d'extrants ou dans un espace auditif. L'ensemble du processus est rapide une fois que le pipeline de base est mis en place et

${ }^{6}$ « Latent See - Emerging Technologies in the Arts », événement d'Anteism Press, 3 octobre 2019, en ligne, https://www.facebook. com/events/498851537579186/, page consultée le 23/01/2021. 
produit souvent des résultats plus nuancés que le codage fixe d'un système réactif. Les appareils d'art intelligents ne sont pas imminents, ils sont arrivés.

En outre, les capteurs de caméra infrarouge de Leap Motion segmentent automatiquement ma main à partir de l'arrière-plan, ils identifient toutes les articulations et les os et cela me permet de simplement fixer une phrase poétique au bout de chaque doigt. Toutes les phrases poétiques proviennent de l'IA. Je n'ai presque rien écrit. J'ai simplement fait un travail d'édition. ${ }^{7}$

À un niveau, la créativité augmentée améliore la capacité et accélère la mise en œuvre. Ce qui m'a pris des semaines en 2005 a été achevé en quelques jours en 2019. La cartographie des contraintes codée en 2005 est maintenant principalement prise en charge par l'apprentissage automatique. L'interpolation est nuancée, multidimensionnelle et automatisée. L'écologie méthodologique de l'art a changé. Les ressources technologiques pour l'automatisation de la perception de base se sont radicalement améliorées. L'interactivité sous-jacente à la quasi-cognition est une nouvelle norme.

Pourtant, en même temps, les choses ont ralenti considérablement! Le travail a augmenté! J'explore la génération de poésie IA depuis cinq ans. Chaque jour, il y a de nouvelles recherches. Chaque mois de nouveaux outils. Trop à apprendre, trop à savoir. Trop de cadres.

Un écosystème explosif de recherche en évolution rapide exige une adaptation et un apprentissage continus. Une certaine lenteur découle de l'excédent, ou peut-être y a-t-il à un point de basculement entre l'abondance et la surcharge. L'exaltation et la fatigue tournent en boucle à travers les cycles. Les tentacules d'un étalement potentiellement contradictoire se déploient vers l'extérieur précipitant la paralysie. Passer au crible des milliers de lignes de poésie générée est laborieux ; passer au crible des millions de lignes est plus que ce qu'un cerveau humain peut s'engager à faire. Comme l'écrit Kyle Booten : " La poétique numérique atteint peut-être une phase si exigeante en maind'œuvre qu'elle peut être mieux comprise comme une forme d'agriculture symbolique. $\|^{8}$

\footnotetext{
${ }^{7}$ David Jhave Johnston. ReRites : Machine Learning Poetry Edited by a Human. Montréal, Anteism, 2019, en ligne, http://glia.ca/ rerites/, page consultée le 23/01/2021.

${ }^{8}$ Kyle Booten, «Harvesting ReRites» dans ReRites - Raw Output / Responses, Montréal, Anteism, 2019, en ligne, https://www. anteism.com/shop/rerites-raw-output-responses-david-jhave-johnston, page consultée le 23/01/2021.
} 


\section{GPT-2: Terminateur ou triomphe?}

Le 14 février 2019, OpenAl a lancé un code de recherche qu'ils ont nommé GPT-2. Lors de son écriture, le GPT-2 était : « formé simplement pour prédire le prochain mot dans 40 Go de texte Internet. En raison de nos préoccupations au sujet des applications technologiques malveillantes, nous ne publions pas ce modèle. ${ }^{9}$ GPT-2 est devenu un mème de la culture pop, une sorte de gène Terminateur du langage humain, un algorithme si puissant que même les modèles capables de générer un langage pratiquement indiscernable de celui de l'humain, ont dû être gardés cachés ou ils auraient pu menacer la capacité de discerner la vérité.

Cependant, même si le modèle complet n'a pas encore été lancé, il existe une version limitée du modèle GPT-2. ${ }^{10}$ Imaginez toutes les règles de syntaxe et les processus statistiques intuitifs qui font partie de la langue, et comprimezles dans un format numérique implacablement restrictif. Le modèle contient une représentation mathématique dense de ce que cet algorithme considère comme le langage.

En avril et mai 2019, j'ai expérimenté avec le modèle 345m GPT-2. Ces expériences ont donné un langage poétique généré par l'IA qui, à mon avis, est historiquement sans précédent dans sa complexité et sa continuité. Comment cela se produit-il? La représentation du langage que contient le modèle peut être peaufinée. Peaufiner, c'est comme polir le noyau dense du modèle avec un idiome spécialisé; en l'exposant à un autre corpus, le modèle est reformé pour une utilisation spécifique. J'ai donc peaufiné GPT-2 avec le corpus de poésie personnalisée que j'avais recueilli pour ReRites.

GPT-2 écrit une sorte de charabia qui pourrait possiblement approcher le niveau de langage d'un enfant de sept ans avec peu d'expérience de vie qui aurait le vocabulaire et la grâce syntaxique d'un conférencier de niveau supérieur quasi expert. Ainsi, alors que la continuité et la conscience incarnée et intégrée sont absentes de larges portions de ce langage artificiel, il existe des capacités dans le langage qui permettent à un praticien (comme moi) d'utiliser le texte généré comme motif pour écrire très rapidement à des niveaux auxquels un individu créatif non augmenté, non amélioré peut rarement aspirer.

\footnotetext{
${ }^{9}$ Alec Radford et al., «Better Language Models and Their Implications», dans OpenAl, 14 février 2019, en ligne, https://openai.com/ blog/better-language-models/, page consultée le 23/01/2021.

${ }^{10}$ Les modèles présentés sur un horaire temporel. Au moment d'écrire ces lignes un $774 \mathrm{mb}$ de taille moyenne a été lancé.
} 
Pendant des millénaires, les poètes, écrivains, musiciens, artistes, inventeurs, théoriciens, et peut-être même les politiciens ont aspiré à des moments d'inspiration incandescente. Des moments culminants. Moments où la pensée se cristallise et dans une rafale de conscience une transformation fondamentale se produit. Souvent, cela coïncide avec un flot de langage. Le processus épiphanique est synonyme de notion moderniste d'inspiration, pour respirer la même atmosphère qu'une muse. Comme les systèmes d'IA capables de générer un langage deviennent de plus en plus ajustables par l'utilisateur, l'écriture et l'inspiration en tant que pratiques incarnées sont appelées à changer. Le GPT-2 m'a convaincu que dans 15 ans, très peu d'auteurs écriront sans l'apport de l'intelligence artificielle.

\section{Pourquoi les changements de capacité sont-ils importants?}

L'intelligence artificielle, comme plusieurs le savent, a fait la transition de formats d'apprentissage supervisés à non supervisés, de systèmes fondés sur des règles d'experts à des réseaux neuronaux. AlphaZeros, ${ }^{11}$ qui ne s'appuie sur aucune donnée humaine, se développe à partir de simples contraintes et génère des représentations denses de règles et de modèles qui sont souvent invisibles et inaccessibles au raisonnement taxonomique humain. En 2005, il était presque impossible de dicter une texte clairement et intelligiblement à un ordinateur. C'était un processus ardu et sujet aux erreurs. En 2019, la première ébauche de ce présent essai a été dictée par la voix à mon téléphone. ${ }^{12}$

Pourquoi les changements de capacité sont-ils importants? Parce que tout comme l'Internet et l'émergence de la blogosphère basée sur le navigateur accessible publiquement, ils ont créé une efflorescence massive de belles explorations créatives, l'espace infonuagique actuel d'IA et de services cognitifs qui permettront une aspiration sauvage similaire à la créativité afin d'exploser à nouveau dans des méditations formelles sans précédent sur l'impact des changements exponentiels technologiques. Les personnes paralysées peuvent marcher. Les aveugles peuvent voir. Dans une certaine mesure, les personnes sourdes avec des implants cochléaires entendent déjà.

\footnotetext{
${ }^{11}$ David Silver, Thomas Hubert, Julian Schrittwieser et Demis Hassabis. «AlphaZero: Shedding new light on the grand games of chess, shogi, and Go.», dans DeepMind, 6 décembre 2018. En ligne, https://deepmind.com/blog/article/alphazero-shedding-new-light-grandgames-chess-shogi-and-go, page consultée le 23/01/2021.

${ }^{12}$ En 2005, dans le but d'obtenir une transcription de la voix au texte, il fallait lire à répétition un long passage de Moby Dick ou d'un autre roman historique jusqu'à ce que le système obtienne une connaissance rudimentaire de votre voix individuelle. En 2019 , la plupart d'entre nous parlent à leurs appareils, les entrainant en répétant quelques mots. Les quelques mots que nous prononçons servent à peaufiner le modèle vocal. Tout comme les modules de langage neurologique, la grammaire générative déterministe évolutive d'un nouveau-né s'accorde à la voix de la mère. Conséquence : les mégadonnées sont intensément puissantes et équivalentes à l'évolution : elles renforcent les connaissances structurelles intégrées à grande échelle ; mais les petites données entraînent un peaufinage unique des modèles de réseau neuronal qui est sur le point de transformer les choses en réactivité.
} 
Imaginez les permutations possibles de l'art! Des jeux livrés directement dans le nerf optique. Un art qui stimule le toucher et l'odorat. Une émotion transmise à des doses nuancées quantifiables. L'art appartiendra au cerveau comme il ne l'a jamais fait auparavant. L'intimité pourra être définie par la place que nous allouons aux autres dans nos pensées et sur le plan physique. Ce sera l'impact positif.

En matière de potentiels impacts négatifs, le monde est perché périlleusement au bord d'un abîme de contrôle tyrannique et absolu et de l'effondrement simultané du substrat de l'écosystème. La transparence de l'individu ne trouve pas écho dans une transparence des processus gouvernementaux et réglementaires formels et fondamentaux ni dans une transparence d'algorithmes capables de pousser, diviser, radicaliser, exploiter et effacer l'autonomie.

Jamais auparavant dans l'histoire de l'humanité les gouvernements et les entreprises n'ont eu autant de pouvoir pour regarder dans notre esprit de façon aussi limpide et impeccable. II n'y a jamais auparavant eu de technologie imminente qui puisse imiter si élégamment l'art : nous faire sentir, nous faire réfléchir, nous faire pleurer, nous faire croire. Véritablement nous fabriquer : par exemple, sans hésitation atteindre et susciter des potentiels d'action au niveau des neurones.

Dans tous les écosystèmes organiques, la cruauté rapace, la prédation, l'exploitation impitoyable et l'égoïsme radical coexistent avec les modes d'être vibratoires empathiques et transcendantaux de la communauté. Les organismes et les espèces de formes diverses s'orientent en groupes, en hardes et en troupeaux autour de l'étreinte instinctive de leurs motivations anatomiques. Cet écosystème métabolique organique ne se transforme pas, il s'effondre en un enchevêtrement désespéré. Les appétits continueront à dominer l'éthique. L'intelligence artificielle deviendra à la fois l'instigatrice d'une renaissance créative et l'aspect intégral de la guerre. Quoi qu'il arrive, les caprices de ce monde finiront par nous confiner alors qu'ils continuent inexplicablement à évoluer.

Il serait sage d'écouter Achille Mbembe : « En tant que référentiel de nos désirs et de nos émotions, de nos rêves, de nos peurs et de nos fantasmes, notre esprit et notre vie psychique sont devenus la principale matière première 
Les implants augmentent simplement le contrôle. Ils ne le créent pas. Dans les entrailles instinctives métaboliques du primate carnivore homo sapiens, percole une volonté de puissance. Incessante, insistante.

\section{Appendice: L'avenir maintenant}

Neuralink est seulement l'un des nombreux laboratoires de recherche ou de sociétés qui opèrent dans le nouvel espace de la mise en contact direct de l'interactivité avec les réseaux synaptiques sinueux et humides de notre cerveau. Le 11 septembre 2019, la page couverture de Nature Machine Intelligence titrait : " Des cerveaux-machine avec interfaces neuronales entièrement portables et sans fils, activés par les électrodes flexibles du cuir chevelu et l'algorithme d'apprentissage profond. $"{ }^{14}$ Le but avoué de l'auteur est d'aider les personnes handicapées à contrôler sans fil un fauteuil roulant électrique, à interagir avec un ordinateur ou à opérer un petit véhicule robot sans avoir à porter un lourd casque d'électrodes ou de composer avec des fils.

L'intelligence artificielle est la clé essentielle du progrès dans le décodage des signaux cérébraux ; c'est une porte techno dans un immense labyrinthe de rapides tirs synaptiques neuronaux. Dans le crâne du mammifère Sapiens des cavernes regardant le monde extérieur inexplicable, ces signaux chatoyants de sensibilité provisoire stimuleront l'interactivité et la communication duplex à distance. Les fils de fibres nano codées dans des capteurs capacitifs insérés ou collés dans les cortices sensoriels et moteurs de notre cerveau liront et écriront l'imagination existante entre les êtres. L'art va exploser et imploser. ${ }^{15}$ Les possibilités d'optimisme sont aussi denses que le sont les débris de catastrophes potentielles.

\footnotetext{
${ }^{13}$ Sindre Bangstad et Torbjørn Tumyr Nilsen, «Thoughts on the Planetary: An Interview with Achille Mbembe», dans New Frame, 5 septembre 2019, en ligne, https://www.newframe.com/thoughts-on-the-planetary-an-interview-with-achille-mbembe/, page consultée le $23 / 01 / 2021$.

${ }^{14}$ Musa Mahmood et al., "Fully Portable and Wireless Universal Brain-Machine Interfaces Enabled by Flexible Scalp Electronics and Deep Learning », dans Nature Machine Intelligence, numéro 9, 11 septembre 2019, pp. 412-422, en ligne, https://doi.org/10.1038/ s42256-019-0091-7, page consultée le 23/01/2021.

15 Soit dit en passant : j'habite actuellement dans le quartier Mile-Ex de Montréal, le même quartier où j'ai commencé à vivre il y a exactement 15 ans, juste après ma résidence à LA CHAMBRE BLANCHE. Mile-Ex est le site d'un boom immobilier lié à l'intelligence artificielle. La femme pour qui j'ai écrit les poèmes d'amour dans Sooth 2005, - SJ qui sait qui elle est, - a vécu pendant plus de 20 ans dans un bâtiment d'entrepôt abandonné avec accès au toit où nous pouvons maintenant voir Element Al, Mila, Samsung, Microsoft, et un assortiment de jeunes pousses à la mode Silicon Valley qui occupent des espaces de bureau suaves avec des spas, des tables de baby-foot et des tables de ping-pong. Cet écosystème d'opportunités et d'embourgeoisement évincera également les artistes (comme SJ et d'autres) et les ouvriers qui habitaient autrefois ce quartier.
} 


\section{Références}

BANGSTAD, Sindre et Torbjørn Tumyr Nilsen. «Thoughts on the Planetary: An Interview with Achille Mbembe», dans New Frame, 5 septembre 2019. En ligne, https://www.newframe.com/thoughts-on-the-planetary-an-interview-with-achille-mbembe/, page consultée le 23/01/2021.

BOOTEN, Kyle. "Harvesting ReRites» dans ReRites - Raw Output / Responses. Montréal, Anteism, 2019. En ligne, https://www.anteism.com/shop/ rerites-raw-output-responses-david-jhave-johnston, page consultée le 23/01/2021.

BREAZEAL, Cynthia L. Designing Sociable Robots. Cambridge et Londres, MIT Press, 2004. 282 p.

DAI, Zihang et al. «Transformer-XL: Attentive Language Models Beyond a Fixed-Length Context» dans Cornell University, Ithaca, 9 janvier 2019. En ligne, https://arxiv.org/abs/1901.02860v2, page consultée le 23/01/2021.

JOHNSTON, David Jhave. ReRites : Machine Learning Poetry Edited by a Human. Montréal, Anteism, 2019. En ligne, http://glia.ca/rerites/, page consultée le 23/01/2021.

JOHNSTON, David Jhave et Stephanie Strickland. ReRites - Raw Output / Responses. Montréal, Anteism, 2019. En ligne, https://www.anteism.com/ shop/rerites-raw-output-responses-david-jhave-johnston, page consultée le 23/01/2021.

"Latent See - Emerging Technologies in the Arts», événement d'Anteism Press, 3 octobre 2019. En ligne, https://www.facebook.com/ events/498851537579186/, page consultée le 23/01/2021.

MAHMOOD, Musa et al. «Fully Portable and Wireless Universal Brain-Machine Interfaces Enabled by Flexible Scalp Electronics and Deep Learning», dans Nature Machine Intelligence, numéro 9, 11 septembre 2019, pp. 412422. En ligne, https://doi.org/10.1038/s42256-019-0091-7, page consultée le 23/01/2021.

Neuralink Launch Event. 2019, fichier numérique, 104 minutes. En ligne, https://www.youtube.com/watch?v=r-vbh3t7WVI\&feature=youtu.be, page consultée le 23/01/2021. 
OORD, Aäron van den et Sander Dieleman. «WaveNet: A Generative Model for Raw Audio» dans DeepMind, 8 septembre 2016. En ligne, https://deepmind.com/blog/wavenet-generative-model-raw-audio/, page consultée le 23/01/2021.

PICARD, Rosalind W. Affective Computing. Cambridge et Londres, MIT Press, 1997. 306 p.

RADFORD, Alec et al. «Better Language Models and Their Implications», dans OpenAl, 14 février 2019. En ligne, https://openai.com/blog/better-language-models/, page consultée le 23/01/2021.

SILVER, David, Thomas Hubert, Julian Schrittwieser et Demis Hassabis. " AlphaZero: Shedding new light on the grand games of chess, shogi, and Go.», dans DeepMind, 6 décembre 2018. En ligne, https://deepmind.com/ blog/article/alphazero-shedding-new-light-grand-games-chess-shogi-andgo, page consultée le 23/01/2021. 